\title{
Anhydrobiosis in yeast: activation effect
}

A.I. Rapoport,

G.M. Khroustalyova and E.N. Kuklina
Institute of Microbiology and Biotechnology,

Latvian University, LV-1586, Riga, Latvia

\author{
Correspondence \\ A.I. Rapoport \\ Laboratory of Cell Biology \\ Institute of Microbiology \\ and Biotechnology \\ Latvian University \\ Kronvald blvd. 4 \\ LV-1586 Riga \\ Latvia \\ Fax: +371 7227925 \\ E-mail: root@rapoport.vernet.lv \\ Presented at the XXIV Annual \\ Meeting of the Brazilian Society \\ of Biochemistry and Molecular \\ Biology, Caxambu, MG, Brasil, \\ May 7-9, 1995. \\ Research supported by the \\ International Science Foundation \\ (No. LBK 000) and the Latvian \\ Scientific Council (No. 853). \\ $\ldots \ldots \ldots \ldots \ldots \ldots \ldots$
}

Received October 1, 1996

Accepted November 5, 1996

..................

\begin{abstract}
Intracellular substances released into the medium during rehydration of dry yeast cells can significantly improve the quality of a synthetic medium. Acceleration of yeast growth in this medium and increased yield of biomass are observed simultaneously. The change in the molecular arrangement of intracellular membranes as a result of the strong dehydration of live organisms is a negative phenomenon that reduces the level of cell viability. However, this phenomenon also represents an adaptive mechanism which facilitates the maintenance of population viability as a whole under extreme environmental conditions.
\end{abstract}

\section{Introduction}

Yeast anhydrobiosis investigations are mainly directed at the detection of the different reconstructions of intracellular structures under conditions of extreme dehydration of living organisms. At the same time they permit us to identify the potential of eukaryotes under unfavorable environmental conditions and to detect previously unknown protective reactions for the maintenance of organism viability, as well as other unusual phenomena. It was in one such study that chromatin condensation in the cell nucleus was detected as one of the most important protective reactions (1). Another important reaction is the segregation of irreversibly damaged cell sites by the plasma membrane (2). Another interesting and unexpected process was the separation of chromatin-containing parts of the nucleus by the nuclear membrane (3). This reaction probably favors the preservation of the minimum bound water content in the cells which is necessary for the maintenance of the structure and conformation of membranes and macromolecules.

When we investigated the anhydrobiosis of the starch-utilizing yeast Endomycopsis (Saccharomycopsis) fibuligera we observed an unusual phenomenon. Using this dehydrated yeast as inoculum under aerobic conditions we obtained higher yields of biomass as well as higher activities of amylolytic enzymes ( $\alpha$-amylase and glucoamylase) than with the use of native yeast as inoculum. We also observed a similar higher intensity of glucose fermentation to alcohol under anaerobic conditions (4).

The objective of the present study was to test the hypothesis of the improvement of the nutrient medium content by the intracellular substances leaking from dried yeast cells during rehydration as a possible mechanism for this unexpected phenomenon.

\section{Material and Methods}

The yeast Saccharomyces cerevisiae 14, 
obtained from the collection of microorganisms of the Laboratory of Cell Biology, Institute of Microbiology and Biotechnology, Latvian University, was grown in nutrient media containing molasses ( $2 \%$ sugar, w/v) or glucose $\left(2 \%\right.$, w/v) plus $0.7 \mathrm{~g} / 1 \mathrm{MgSO}_{4}, 0.5$ $\mathrm{g} / \mathrm{l} \mathrm{NaCl}, 1.0 \mathrm{~g} / \mathrm{l}\left(\mathrm{NH}_{4}\right)_{2} \mathrm{SO}_{4}, 1.0 \mathrm{~g} / \mathrm{K}_{2} \mathrm{HPO}_{4}$, and $0.13 \mathrm{~g} / \mathrm{KH}_{2} \mathrm{PO}_{4}$ in flasks kept on a rotary shaker at $30^{\circ} \mathrm{C}$.

The cells were dehydrated at $37^{\circ} \mathrm{C}$ by the convective method using a laboratory model of fluidized bed drier. To obtain dead cells, after the normal dehydration procedure we placed the dry cells in an oven at $105^{\circ} \mathrm{C}$ for $30 \mathrm{~min}$. The experiment was carried out as follows: dry viable (variant 1 ) and dry dead (variant 2) yeast cells were rehydrated in 10 $\mathrm{ml}$ distilled water for $15 \mathrm{~min}$ at $37^{\circ} \mathrm{C}$. We then inoculated compressed yeast (100 mg dry weight) or a suspension of rehydrated viable yeast cells (100 mg dry weight) into flasks containing $100 \mathrm{ml}$ of glucose or molasses medium. We then added rehydration medium from the first or second variant of the experiment to some flasks and the same amount of distilled water to control flasks. All flasks were transferred to the shaker and cultured as described earlier. Absorbance at $660 \mathrm{~nm}$ was determined at 4-h intervals, and biomass yield was determined using the calibration curve for our preliminary culture.

\section{Results and Discussion}

Some hypotheses may be raised to explain such an unusual phenomenon as higher activity of amylolytic enzymes and higher accumulation of biomass under aerobic conditions as well as higher levels of glucose fermentation to alcohol when dehydrated cells rather than the normally used native yeast Endomycopsis fibuligera were employed as the inoculum (4). One possibility is that some genetic changes may occur in the cells during the dehydration process although it is difficult to justify the reproducibility of this phenomenon in each experi- ment during the drying of Endomycopsis fibuligera. Another possibility is that some yeast cell systems may be activated during the drying process, leading to an enhanced multiplication rate during their subsequent culture. This is an interesting possibility, especially if we consider that we have previously shown the activation and synthesis of a number of enzymes during the drying process, among them acid phosphatase, ribonuclease and others $(5,6)$. An enhanced specific growth rate of viable dry yeast during drying has also been reported (7). However, this would explain changes for only some generations but not for the entire period of yeast culture. In our opinion, a much more likely hypothesis is that changes in the molecular arrangement of the plasma membrane are the major factor determining this effect. As shown earlier, these changes are responsible for the highly significant increase of plasma membrane permeability during the rehydration of dry organisms. Different intracellular substances ranging from ions and nucleotides to rather large molecules can leak into the surrounding medium under these conditions (8-11). At the same time, varying numbers of cells irreversibly damaged during the dehydration procedure are always present in dry cell populations side by side with anhydrobiotic (viable) organisms. Of course some cells can also lose viability during the rehydration stage because this step is known to cause significant stress for damaged organisms, and when repair conditions are inadequate, part of the population may be killed. It is clear that larger quantities of intracellular substances can leak into the medium from dead cells than from viable cells. Thus, it is possible that some of these compounds can essentially improve the quality of the nutrient medium which will be used for reactivation of viable organisms, providing the conditions for accelerated restoration and subsequent growth and multiplication of the latter. This hypothesis was tested in the present study. 
Many methodologic problems were faced in the present investigation using the yeast Endomycopsis fibuligera. For example, it was rather difficult to determine the rate of viability of mycelial forms of this yeast, differences in the plasma membrane permeability of mycelial and cellular forms of this yeast were detected, and the level of lysis that could occur at the time when the mycelial forms were transformed into cellular forms and during their subsequent culture was difficult to determine. For these reasons, we decided to use Saccharomyces cerevisiae, which also presents some other advantages. The use of this yeast, in addition to eliminating the problems mentioned above, also permitted us to determine whether this phenomenon occurs in a similar manner in any yeast species. Indeed, the major investigations of different aspects of yeast anhydrobiosis have been carried out on Saccharomyces cerevisiae.

In the first set of experiments dried Saccharomyces cerevisiae yeast was rehydrated, reactivated and cultured in nutrient medium containing molasses. The results obtained are illustrated in Figure 1. When three samples of dry yeast were compared, a certain reproducibility was observed. Dry yeast cells depleted of intracellular substances that had leaked during rehydration showed the worst growth rate and produced the lowest biomass yield. Both of these parameters were slightly better in the sample of dry yeast which was not depleted of leaked substances. The best results were obtained when we added the rehydration liquid obtained by rehydrating dead dry yeast to the nutrient medium. Furthermore, after $48 \mathrm{~h}$ of dry yeast cultivation under these conditions we observed higher biomass yields compared to the experiment in which the normally used native yeast inoculum was cultured in control (standard) molasses medium without additives.

The results obtained in experiments in which native yeast was used as inoculum

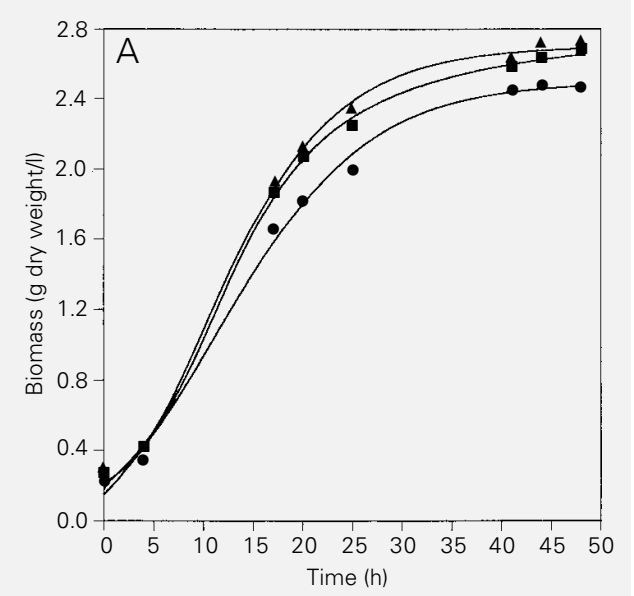

Figure 1 - Biomass accumulation during culture of Saccharomyces cerevisiae in molasses medium using dehydrated yeast (A) and native yeast $(B)$ as inoculum. $\bullet$ Medium with water; medium with liquid obtained by rehydration of viable dry yeast: $\boldsymbol{\Delta}$, medium with liquid obtained by rehydration of dead dry yeast.

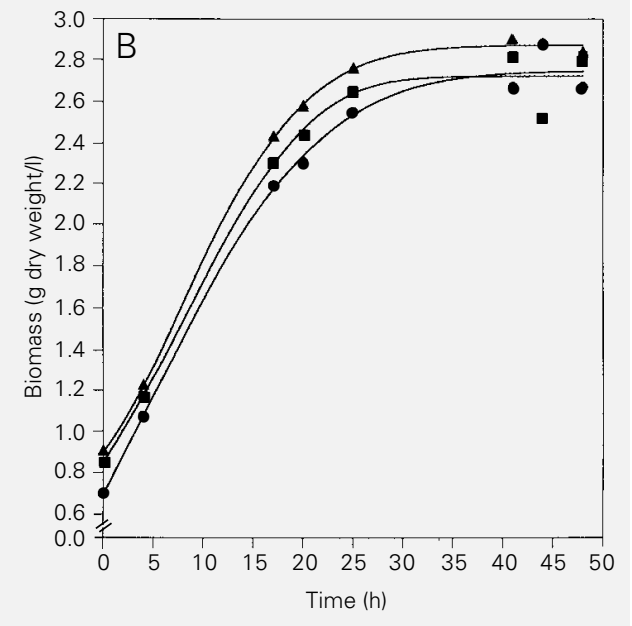

and was cultured in molasses medium with or without different additives were similar (Figure 1). It can be seen that the addition of rehydrating fluids to the nutrient medium resulted in accelerated growth and in a higher biomass yield.

It is well known that molasses medium is one of the best for Saccharomyces cerevisiae since it contains almost all the components necessary for growth. Therefore, we decided to determine whether the same effect occurred when synthetic medium containing glucose as the carbon source was used for yeast growth. The results of these experiments are shown in Figure 2. As expected, the effect of the addition of rehydration liquid to the medium was highly significant. Also, if we used as additive the rehy- 
Figure 2 - Biomass accumulation during culture of Saccharomyces cerevisiae in synthetic medium using dehydrated yeast (A) and native yeast $(B)$ as inoculum. $\bullet$, Medium with water; $\mathbf{\square}$, medium with liquid obtained by rehydration of viable dry yeast; $\boldsymbol{\Lambda}$, medium with liquid obtained by rehydration of dead dry yeast.
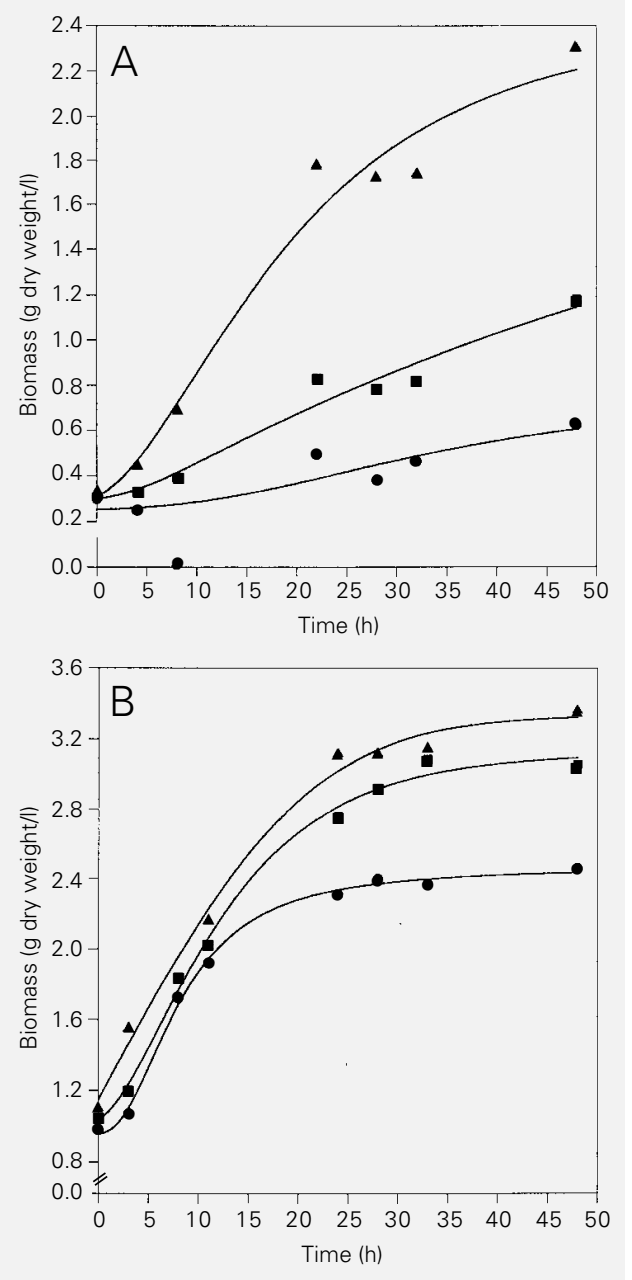

dration liquid obtained from the rehydration of dead dry cells, the growth rate of viable cells exceeded the growth rate of native cells grown in the same medium without additive. Thus, the final biomass yield obtained by culturing dry and reactivated yeast in medium supplemented with the liquid obtained by rehydrating dead dry cells was significantly higher (3-4 times) than the yield obtained when the same cells were grown in nutrient medium without additives.

These experiments clearly showed that the intracellular substances that leaked from dry cells during rehydration greatly improved the content of the nutrient medium. This was important not only for "weakened" dry cells, as expected, but also for native (control) organisms.

In view of the higher biomass yields obtained when native yeasts were cultured in synthetic medium supplemented with rehydration liquid than when they were cultured in molasses medium, we may propose that synthetic medium supplemented with these additives is more "balanced" for Saccharomyces cerevisiae than standard molasses medium.

Thus, the present results confirm our proposal that the unusual "growth activation effect" observed in yeasts reactivated after anhydrobiosis may be linked to the phenomenon of "self-improvement" of the nutrient medium by the leakage of substances from dry yeasts during rehydration. The reason for the more significant effect observed for the yeast Endomycopsis fibuligera compared to Saccharomyces cerevisiae may be that, even if molasses medium is highly appropriate for $S$. cerevisiae, it is not so balanced for Endomycopsis fibuligera, which is known to produce amylolytic enzymes $(\alpha$-amylase and glucoamylase).

In this case this medium could be significantly improved (especially for Endomycopsis fibuligera) as the result of leakage of certain intracellular substances from dry yeast cells during their rehydration. Of course it would be very interesting to identify the factors responsible for this effect.

Although the results obtained in the present study confirm one of the mechanisms of the "activation effect" proposed by us, we cannot exclude the possibility that other active factors may also exist. An additional interesting aspect is the effect of changes in the structural and functional characteristics of membranes that occur during cell dehydration on cell viability. At present, we are working with two hypotheses about the possible changes in the molecular organization of intracellular membranes occurring during the dehydration of living organisms that lead to their transfer to the state of anhydrobiosis. According to the first hypothesis, intracellu- 
lar membrane lipids pass from the lamellar phase to the hexagonal phase II $\left(H_{\mathrm{II}}\right.$-phase) during dehydration $(8,12,13)$. However, since we are talking about the conditions that guarantee the preservation of organism viability, we must assume that such drastic intracellular membrane changes only occur at very small sites on the cell membrane. Since the $H_{\text {II }}$-phase of lipids has not been detected in dry seeds or pollen (14) despite intensive biophysical investigation, we may propose the second hypothesis according to which there is a transition of membrane lipids from the liquid crystalline phase to gel during the dehydration of yeast cells (14). Several investigators have proposed that this hypothesis applies to cases in which the relative humidity of pollen is higher than $0.05 \mathrm{~g}$ water per $1 \mathrm{~g}$ dry substance $(>5 \%)$ and that the existence of the $H_{\mathrm{II}}$-phase is possible in the presence of a higher level of desiccation (15). Regardless of which of these hypoth- eses is more plausible, all anhydrobiosis investigators agree that one of the factors responsible for the maintenance of dry organism viability is the maximum preservation of the initial molecular organization of intracellular membranes. However, the present results show that more complicated relationships seem to be at work here. It seems that some break in plasma membrane permeability as a result of certain changes in molecular organization may have a positive effect also on viable organisms after dehydration-rehydration procedures. Thus, an interesting situation arises: on the one hand, damage to the molecular organization of intracellular membranes or a break in these membranes is very dangerous for individual cells in the microbial population, but on the other hand, these changes may alsorepresent a protective reaction for the maintenance of the viability of the population as a whole under extreme environmental conditions.

\section{References}

1. Rapoport Al \& Kostrikina NA (1973). Cytological investigation of an anabiosis state of yeast organisms. Proceedings of the Academy of Sciences of the USSR, 5: 770-773 (in Russian).

2. Rapoport Al (1973). Rejection of areas of damaged cytoplasm by microorganisms in a state of anabiosis. Microbiology, 42: 317-318.

3. Rapoport Al, Birjusova VI \& Meissel MN (1973). The structure of the yeast cell nucleus in an anabiotic state. Reports of the Academy of Sciences of the USSR, 213: 708-710 (in Russian).

4. Rapoport Al, Dreimane MA \& Beker ME (1986). Influence of the dehydration process on Endomycopsis fibuligera yeasts. Microbiology, 55: 542-545.

5. Rapoport Al \& Beker ME (1978). Increased intracellular activity of acid phosphatase on desiccation of the yeast, Saccharomyces cerevisiae. Microbiology, 47: 136-137.
6. Rapoport Al \& Beker ME (1986). Ribonucleic acid degradation during dehydration of yeast cells. Microbiology, 55: 689691.

7. Meledina TV, Vitrinskaya AM \& Soboleva GA (1978). Some peculiarities of metabolism and multiplication of yeast Saccharomyces cerevisiae upon their reactivation. Mikrobiologiya, 41: 51-55 (in Russian).

8. Beker ME \& Rapoport Al (1987). Conservation of yeasts by dehydration. Advances in Biochemical Engineering/Biotechnology, Springer-Verlag, Berlin, 35: 127-171.

9. Novichkova AT \& Rapoport Al (1984). State of the intracellular pool of free amino acids in dehydrated yeast organisms. Microbiology, 53: 1-4

10. Rapoport Al, Markovskii AB \& Beker ME (1982). Increase in the permeability of intracellular membranes on dehydration and rehydration of Saccharomyces cerevisiae yeast. Microbiology, 51: 707-710.
11. Rapoport Al, Pomoshchnikova NA Fateeva MV, Nikitina TN \& Meissel MN (1981). Nucleic acids and resistance of yeast cells to dehydration. Microbiology, 50: 163-165.

12. Luzzati $\vee \&$ Husson $F$ (1962). The structure of the liquid-crystalline phases of lipid-water systems. Journal of Cell Biology, 12: 207-219.

13. Simon EW (1974). Phospholipids and plant membrane permeability. New Phytologist, 73: 377-420.

14. Crowe JH, Hoekstra FA \& Crowe LM (1989). Membrane phase transitions are responsible for imbibitional damage in dry pollen. Proceedings of the National Academy of Sciences, USA, 86: 520-523.

15. Hoekstra FA, Crowe JH \& Crowe LM (1992). Germination and ion leakage are linked with phase transitions of membrane lipids during imbibition of Typha latifolia pollen. Plant Physiology, 84: 2934. 